\title{
Methods of estimating hydraulic and transport parameters for the unsaturated zone
}

\author{
S MISHRA and J C PARKER \\ Centre for Environmental and Hazardous Materials Studies, Virginia \\ Polytechnic Institute and State University, Blacksburg, Virginia 24061, \\ USA
}

MS received 15 July 1989

\begin{abstract}
Methods of estimating hydraulic and transport parameters for the unsaturated zone are presented. Two approaches to parameter estimation are discussed. The first approach is based on inversion of the governing initial-boundary value problem for unsaturated flow and/or transport using data from transient experiments. The second approach utilizes particle size distribution data to obtain estimates of soil hydraulic properties. Example applications of the two different approaches are presented and their advantages and limitations are discussed. Procedures for identifying and quantifying sources of uncertainty in parameter estimates are examined. Ongoing research on the scale-up of parameters for applications in large-scale numerical simulations is reviewed.
\end{abstract}

Keywords. Hydraulic parameters; transport parameter; unsaturated zone; uncertainty in parameter estimate; large-scale numerical simulations.

\section{Introduction}

Recent concerns with groundwater contamination and the associated use of numerical simulations of unsaturated flow and transport for accurate definition of pollutant sources have motivated the development of better model calibration practices. Modelling of water flow and chemical transport through the unsaturated zone requires knowledge of both hydraulic and transport parameters of the medium. Hydraulic properties define the relationship between conductivity $(K)$, volumetric water content $(\theta)$ and capillary head $(h)$; transport properties are considered to be those which characterize the tendency of a solute to spread as it moves through the subsurface. In practice, such properties are often represented via simple parametric models based on physical and/or empirical considerations.

This paper reviews two different methods for estimating hydraulic and transport parameters for the unsaturated zone. The first method uses data from transient unsaturated flow and tracer experiments to invert the governing initial-boundary value problem and estimate hydraulic and transport parameters. The second method 
uses particle size distribution (PSD) data in conjunction with a quasi-physical predictive model to provide estimates of soil hydraulic properties.

Since predictions of unsaturated flow and transport are sensitive to errors in model parameters, it is necessary to provide estimates of parameter uncertainty for assessing the reliability in numerical model predictions. A related issue is the development of procedures to scale-up parameters obtained at the laboratory and/or core scale for application in field-scale simulations of flow and transport. Current modelling practices with respect to these questions will be briefly reviewed.

\section{Parameter estimation from inversion analysis}

Given a parametric model of the physical system and values of the model parameters, the prediction of system response (output) due to any stimulus (input) is referred to as the direct problem. Conversely, the estimation of model parameters given the parametric system model and an input-output relationship is known as the inverse problem. A typical inverse problem solution scenario involves guessing values of the model parameters, solving the direct problem with this set of parameters to calculate the predicted system response, and adjusting model parameters until deviations between observed and model-predicted responses are minimized.

Conventional methods for determining soil hydraulic and transport properties are based on laboratory or field experiments which use simple analytical or semi-analytical solutions to the direct problem. Such methods, however, generally cannot handle arbitrary initial and/or boundary conditions, nor can they explicitly account for spatially variable porous medium properties. An alternative calibration approach is to use experimental data in conjunction with a numerical code for solving the direct problem, and an optimization algorithm for solving the inverse problem and estimating parameters. Such a simulation-optimization methodology provides greater flexibility in experimental design and ensures that the estimated parameters are optimal in some specified sense.

There is a growing body of literature dealing with the application of inversion analysis to subsurface hydrologic systems. Yeh (1986), Carrera \& Neuman (1986), and Wagner \& Gorelick (1987) have reviewed parameter estimation techniques for saturated flow and/or transport models. Application of inverse methods for the estimation of unsaturated flow and/or transport parameters has been described by Zachmann et al (1982), Hornung \& Messing (1982), Parker \& van Genuchten (1984), Kool et al (1985, 1987), Jury \& Sposito (1985), Kool \& Parker (1988) and Mishra \& Parker (1989a). In this section, we briefly present a general formulation for inversion analysis of coupled unsaturated flow and transport and an example application of the methodology.

\subsection{Direct problem for unsaturated flow and transport}

The governing equation for one-dimensional vertical transient water flow, assuming negligible air pressure gradient, is described by Richards' equation

$$
C \frac{\partial h}{\partial t}=\frac{\partial}{\partial x}\left\{K \frac{\partial h}{\partial x}-K\right\}
$$

where $h$ is pressure head, $x$ is depth, $t$ is time, $C=\mathrm{d} \theta / \mathrm{d} h$ is the soil-water capacity 
with $\theta$ the volumetric water content, and $K$ is the hydraulic conductivity. Unsaturated hydraulic properties, $\theta(h)$ and $K(\theta)$, are parameterized via the van Genuchten (1980) formulation, where the retention function, $\theta(h)$, is represented by

$$
S_{e}=\left(\theta-\theta_{r}\right) /\left(\theta_{s}-\theta_{r}\right)=\left\{1+(\alpha h)^{n}\right\}^{-m},
$$

and the conductivity function, $K(\theta)$, is given by

$$
K=K_{s} S_{e}^{1 / 2}\left\{1-\left(1-S_{e}^{1 / m}\right)^{m}\right\}^{2}
$$

Here $K_{s}=K\left(\theta_{s}\right)$ is the saturated hydraulic conductivity, $S_{e}$ is effective saturation, $\theta_{s}$ is saturated water content, $\theta_{r}$ is residual water content, $\alpha$ and $n$ are van Genuchten (VG) model parameters, and the exponent $m=1-1 / n$. The set of hydraulic parameters, therefore, is given by $\alpha, n, \theta_{r}, \theta_{s}$, and $K_{s}$.

The governing equation for transport of a conservative solute is given by the one-dimensional convection-dispersion equation

$$
\frac{\partial(\theta c)}{\partial t}=\frac{\partial}{\partial x}\left\{D \theta \frac{\partial c}{\partial x}-q c\right\},
$$

where $c$ is the solution concentration, $q$ is the darcian flux density and $D$ is a hydrodynamic dispersion coefficient given by the following empirical expression

$$
D=\varepsilon v=v /\left[\left(1 / \varepsilon_{x}\right)+(1 / \beta x)\right] .
$$

Here $v=q / \theta$ is the average pore water velocity, $\varepsilon$ is the dispersitivity, $\varepsilon_{\infty}$ is an asymptotic dispersivity, and $\beta$ is a dispersion scale factor. When $\beta \rightarrow \infty,(5)$ reduces to the classical model of constant dispersivity (i.e., $\varepsilon=\varepsilon_{x}$ ), whereas when $\varepsilon_{x} \rightarrow \infty$, a linear increase of dispersivity with distance is modelled (i.e., $\varepsilon=\beta x$ ). The hyperbolic scale-dependent dispersion formulation used above is consistent with recent observations from theoretical studies (e.g., Dagan 1986, Gelhar 1986) and from field experiments (e.g., Jury et al 1982, Gelhar et al 1985, Freyberg 1986), which indicate that dispersivity increases linearly with the distance of solute plume travel and approches an asymptotic value only when the mean distance of travel is much greater than the average length scale of porous medium inhomogeneities. From (5) then, the set of transport parameters is given by $\varepsilon_{x}$ and $\beta$. Solving the direct problem for coupled unsaturated flow and transport thus requires a knowledge of the five hydraulic parameters $\left(\alpha, n, \theta_{s}, \theta_{r}, K_{s}\right)$, the two transport parameters $\left(\varepsilon_{x}, \beta\right)$, and appropriate initial and boundary conditions.

\subsection{Formulation and solution of the inverse problem}

The objective of the inverse problem is to estimate unknown model parameters by minimizing some function of the difference between observed system attributes (e.g., pressure heads, water contents, concentrations) and model-predicted responses. In general, this involves solving a weighted least-squares problem formulated as follows

$$
\min \Phi=\left(\mathbf{q}^{*}-\tilde{\mathbf{q}}\right)^{\mathrm{T}} \mathbf{V}^{-1}\left(\mathbf{q}^{*}-\tilde{\mathbf{q}}\right)
$$

where $\mathbf{q}^{*}=\mathbf{q}^{*}(x, t)$ represents the vector of observations whose elements are pressure head, water content, and/or concentration measurements, and $\tilde{\mathbf{q}}=\tilde{\mathbf{q}}(x, t ; \mathbf{b})$ is the corresponding vector of predicted model response for a given vector of unknown parameters, $\mathbf{b}$. The matrix, $\mathbf{V}$, is the measurement error covariance matrix which is generally unknown. In practice, reasonable results have been obtained by assuming 
that measurements of a certain type have a common variance proportional to the measurement means, and then normalizing the ratios of error variances such that measurements with the lowest mean are assigned unit variance (Kool \& Parker 1988; Mishra \& Parker 1989a).

Since transient unsaturated flow and transport are coupled processes, hydraulic and transport parameters may be estimated in principle using either of two alternative approaches. In the sequential methodology, pressure head and water content data are first used to invert the flow equation and estimate the hydraulic parameters. These estimates are then used together with concentration data to invert the transport equation. The simultaneous methodology uses pressure head, water content and concentration data concurrently to simultaneously estimate hydraulic and transport parameters. Since soil hydraulic properties influence solute transport, using concentration measurements to condition estimates of hydraulic parameters (as in the simultaneous method) should result in more optimal parameter estimates. This has been verified for hypothetical data sets by Mishra \& Parker (1989a).

The nature of the problem for coupled unsaturated flow and transport necessitates adopting an iterative approach for solving the inverse problem. Beginning from an initial parameter vector, $b^{0}$, the procedure involves computing a correction vector, $\Delta b$, at every iteration, $i$, such that

$$
\boldsymbol{\Phi}\left(\mathbf{b}^{i}+\Delta \mathbf{b}^{i}\right) \leqslant \boldsymbol{\Phi}\left(\mathbf{b}^{i}\right)
$$

until changes in the objective function, $\Phi$, and/or elements of the correction vector, $\Delta b$, are significantly small. The Levenberg-Marquardt modification of the GaussNewton minimization algorithm (Beck and Arnold 1977) is used to calculate $\Delta \mathbf{b}$ by solving the nonlinear system

$$
\mathbf{J}^{\mathrm{T}} \mathbf{W} \mathbf{J} \Delta \mathbf{b}=-\mathbf{J}^{\mathrm{T}} \mathbf{W} \mathbf{e}
$$

where $\mathbf{e}=\left(\mathbf{q}^{*}-\tilde{\mathbf{q}}\right)$ is the vector of residuals, $W=V^{-1}$ is the weighting matrix, and $\mathbf{J}$ is the Jacobian (sensitivity) matrix whose elements are given by

$$
J_{i j}=-\partial \tilde{q}_{i} / \partial b_{j} \simeq\left[\tilde{q}\left(x_{i}, t_{i} ; b_{j}+\delta b_{j}\right)-\tilde{q}\left(x_{i}, t_{i} ; b_{j}\right)\right] / \delta b_{j},
$$

with the forward difference increment taken to be $\delta b_{j}=0.01 b_{j}$.

\subsection{Evaluation of parameter uncertainty}

Estimates of parameter uncertainty can be obtained from the a posteriori parameter covariance matrix. A first-order approximation of this matrix can be formed from the final least-squares error, elements of the weighting matrix (i.e., the inverse of the a priori parameter covariance matrix) and the final Jacobian matrix (e.g., Beck \& Arnold 1977). Diagonal elements of the parameter covariance matrix contain the individual parameter variances, whose square root is simply the standard error of estimate. The off-diagonal elements represent the covariance terms, and upon being normalized by the individual parameter variances, they reflect the correlation between parameters. Parameter confidence limits can also be determined from individual parameter variances, albeit approximately, using $t$-statistics (Kool \& Parker 1988).

\subsection{Example application}

This example demonstrates the estimation of parameters for coupled unsaturated flow and transport for a hypothetical homogeneous system. In order to generate a 
Table 1. Actual and estimated parameters for hypothetical coupled flow and transport problem.

\begin{tabular}{lcccccccc}
\hline Parameter & $\begin{array}{c}\text { Actual } \\
\text { value }\end{array}$ & $\begin{array}{l}\text { Initial } \\
\text { guess }\end{array}$ & $\begin{array}{c}\text { Final } \\
\text { value }\end{array}$ & $\begin{array}{c}\text { Standard } \\
\text { error }\end{array}$ & & & \multicolumn{4}{c}{ Correlation matrix } \\
\hline$x\left(\mathrm{~cm}^{-1}\right)$ & 0.04 & 0.025 & 0.0393 & 0.0004 & 1.000 & & $K_{s}$ & $\varepsilon_{\infty}$ \\
$n$ & 1.60 & 1.321 & 1.5922 & 0.0092 & -0.189 & 1.000 & \\
$K_{s}\left(\mathrm{~cm} \mathrm{~h}^{-1}\right)$ & 1.00 & 0.430 & 1.1387 & 0.0065 & 0.181 & -0.880 & 1.000 & \\
$\delta_{x}(\mathrm{~cm})$ & 1.00 & 0.520 & 1.1192 & 0.0347 & -0.168 & -0.274 & 0.273 & 1.000 \\
\hline
\end{tabular}

synthetic data set consisting of "measurements" of concentration, water content and pressure head, the direct problems, (1) and (4), were solved for prescribed initial and boundary conditions and constitutive relations. The experiment simulated was a ponded infiltration/redistribution sequence in a soil column extending to a water table at $200 \mathrm{~cm}$ depth. Assuming initially hydrostatic conditions, the surface was ponded with a tracer solution of unit concentration for $12 \mathrm{~h}$ after which water was allowed to redistribute for a period of 3 days. This wetting/drying sequence was repeated one more time with tracer-free water added during the second cycle. Water contents and concentrations were assumed to be recorded at depths of $5,15,25,35$, $50,70 \mathrm{~cm}$ and pressure head measurements were taken at $15 \mathrm{~cm}$. These measurements were assumed to be taken only once during each ponding/redistribution period, i.e. at $5,36,90$ and 144 hours. The data set was perturbed by adding an $N\left(0, \sigma^{2}\right)$ error term to the exact measurements. Assumed error standard deviations for different types of measurements were $\sigma_{h}=2 \mathrm{~cm}, \sigma_{\theta}=0.02, \sigma_{c}=0.02$.

Data for this problem were generated using non-hysteretic soil hydraulic properties and a constant dispersivity and were analysed using the same assumptions. Assumed values of the parameters were: $\alpha=0.04 \mathrm{~cm}^{-1}, n=1.6, \theta_{r}=0.08, \theta_{s}=0.42$, $K_{s}=1 \mathrm{~cm} \mathrm{~h}^{-1}$ and $\varepsilon_{\infty}=1 \mathrm{~cm}$. For the inversion, values of $\theta_{\mathrm{s}}$ and $\theta_{r}$ were assumed to be known exactly, leaving $\alpha, n, K_{s}$ and $\varepsilon_{\infty}$ as the unknown parameters. Table 1 shows the actual parameter values, initial guesses, final parameter estimates, standard errors of estimate and the parameter correlation matrix. Note the agreement between final estimates and actual values as well as the relatively small standard errors.

Figure 1 shows a comparison between 'observed' water content and concentration profiles and those predicted using the final parameter estimates at 36 and $144 \mathrm{~h}$. Note the excellent agreement between observed and predicted values. Similar agreement was also obtained at sampling times of 5 and $90 \mathrm{~h}$. Although not presented here, a discussion of the methodology for converting uncertainties in the parameter estimates (as given in table 1) into error intervals in numerical model predictions of unsaturated flow and/or transport may be found elsewhere (Mishra \& Parker 1989b).

\section{Parameter estimation from PSD data}

The rationale for estimating soil hydraulic properties from particle size distribution (PSD) data is that soil textural information is in general more readily available than static or dynamic hydraulic data. Methods have been proposed for computing soil-water retention relations from PSD data using models with quasi-physical bases (e.g., Arya \& Paris 1981, Haverkamp \& Parlange 1986), or using regression equations (e.g., McCuen et al 1981, Rawls \& Brakenseik 1985). Methods for estimating saturated conductivity, $K_{s}$, from PSD data are generally based on use of the Kozeny-Carman 

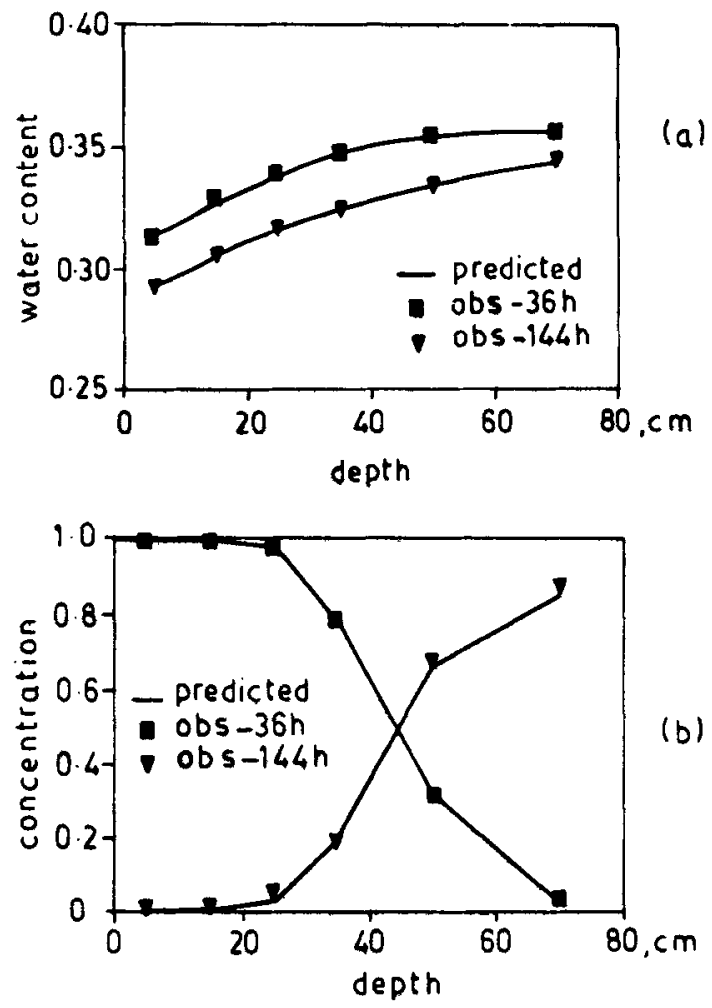

Figure 1. Comparison of observed and predicted water content profile (a) and concentration profile (b).

equation or variations thereof (Dullien 1979). In this section we describe briefly the Arya and Paris (AP) model for predicting soil hydraulic properties from PSD data, and a modified Kozeny-Carman equation for estimating $K_{s}$ from PSD statistics and porosity (Mishra et al 1989).

\subsection{Use of a quasi-physical pore structure model}

The basic premise of this approach is that the soil-water retention function, $\theta(h)$, reflects an underlying pore size distribution which can be inferred from PSD data using a model such as that presented by Arya \& Paris (1981). The Arya and Paris (AP) method involves dividing the particle size cummulative distribution function (CDF) into a number of fractions, assigning a pore volume and a volumetric water content to each fraction, and then computing a representative pore radius and a corresponding capillary head. In our implementation of the AP procedure (Mishra et al 1989), the particle size CDF is sub-divided into 100 fractions, with the pore volume associated with each size fraction, $i$, given by

$$
V_{p_{i}}=e W_{i} / \rho_{s},
$$

where $V_{p_{i}}$ is the pore volume and $W_{i}$ is the solid mass per unit sample mass, $\rho_{s}$ is the particle density, $\phi$ is the porosity, and $e=\phi /(1-\phi)$ is the void ratio. The volumetric water content is computed by progressively filling the pore volumes generated by the size fractions, and is given as

$$
\theta_{i}=\sum_{j=1}^{i} \rho_{b} V_{p j}
$$


where $\rho_{b}$ is the sample bulk density. A relationship between pore and particle radii is obtained by assuming that solid particles are spherical in shape and the effective pore space is cylindrical. The total solid volume in each size fraction is given by

$$
V_{s_{i}}=W_{i} / \rho_{s}=4 \pi n_{s_{i}} R_{i}^{3} / 3,
$$

and the total pore volume is estimated as

$$
V_{p i}=e W_{i} / \rho_{s}=\pi l_{i} r_{i}^{2} \text {. }
$$

Here $n_{s_{i}}$ is the number of spherical particles, $R_{i}$ is the mean particle radius, $r_{i}$ is the mean pore radius, and $l_{i}$ is the total pore length. The total pore length is approximated as the number of particles that lie along the total pore path times the length contributed by each particle, appropriately modified for non-sphericity of the actual soil particles which tends to increase the total pore length, and is given as $l_{i}=2 R_{i}\left(n_{s_{i}}\right)^{t}$, where $\tau$ is a tortuosity exponent. An expression for the mean pore radius, corresponding to $\theta_{i}$, is then obtained as

$$
r_{i}=0.8165 R_{i}\left\{e\left(n_{s_{i}}\right)^{1-\tau}\right\}^{1 / 2} .
$$

Arya \& Paris (1981) suggested an average value of 1.38 for the exponent, $\tau$. In a previous study of 250 soil samples (Mishra et al 1989), $\tau$ was found to be well described by $\tau=\exp \left[0.183 \sigma_{\ln (d)}\right]$, where $\sigma_{\ln (d)}$ is the standard deviation of the CDF of the logarithm of particle diameters.

After the pore radii are calculated for each of the size classes corresponding to a particular volumetric water content, the equivalent air-water capillary pressure, $h_{i}$, can be obtained from

$$
h_{i}=(2 \gamma) /\left(\rho_{w} g r_{i}\right)
$$

where $\gamma$ is the surface tension of water, $\rho_{w}$ is the density of water, and $g$ is acceleration due to gravity. Once the complete $\theta(h)$ functional relationship is generated in the manner described above, it can be fitted to the van Genuchten (VG) model, (2), using nonlinear regression methods to estimate the soil-water retention parameters $\left(\alpha, n, \theta_{r}\right)$. An alternative to the vG formulation for representing soil hydraulic properties is the Brooks-Corey (BC) constitutive relation model (Brooks \& Corey 1964). An empirical method for relating VG and BC model parameters (by matching the area under the $S_{e}-\ln (h)$ curves) has been proposed by Lenhard et al (1989) recently.

\subsection{Estimation of saturated conductivity}

We represent saturated conductivity, $K_{s}$, by the expression

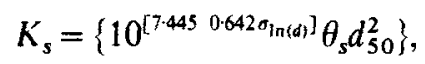

where $K_{s}$ is expressed in $\mathrm{cm} \mathrm{h}^{-1}, d_{50}$ is the median particle diameter in $\mathrm{cm}$, and $\theta_{\mathrm{s}}$ is the saturated water content (assumed to be the same as porosity, $\phi$ ). This equation was obtained by calibrating a data set of 250 soil samples for which measurements and/or estimates of $K_{s}, d_{50}, \theta_{s}$ and $\sigma_{\ln (d)}$ were available (Mishra et al 1989) and is only intended to provide order-of-magnitude estimates.

\subsection{Evaluation of uncertainty}

Parameter estimates obtained from the AP method as discussed above may be in error 
Table 2. Particle size distribution data for example soil.

\begin{tabular}{llllllll}
\hline \multicolumn{7}{c}{ Particle size distribution*, mm } \\
\cline { 1 - 5 } $2.0-$ & $1.0-$ & $0.5-$ & $0.25-$ & 0.10 & $0.05-$ & $\ll$ & $\begin{array}{c}\text { Saturated } \\
\text { water content }\end{array}$ \\
\hline 8.0 & 0.5 & 0.25 & 0.10 & 0.05 & 0.002 & 0.002 & \\
\hline 8.7 & 5.0 & 2.8 & 5.8 & 7.1 & 54.4 & 16.2 & 0.43 \\
\hline
\end{tabular}

From Topp (1971)

* Percentage material in designated size classes

due to several reasons. The uncertainty in the calibrated value of the tortuosity exponent, $\tau$, leads to uncertainty in the AP model generated $\theta(h)$ data, which is further compounded by the possibility of a lack of fit between the VG model and the $\theta(h)$ data. The estimation of $K_{s}$ from the modified Kozeny-Carman equation is also likely to be in error because of the uncertainty in estimating PSD statistics and porosity. Methods for quantifying such uncertainties have been described in detail by Mishra et al (1989) using a first-order error analysis procedure to estimate individual parameter estimation variances, as well as a parameter covariance matrix. As described in the previous section, the elements of the covariance matrix can be used to calculate the standard error in each parameter estimate and the corresponding parameter correlation matrix.

\subsection{Example application}

The methodology described above is applied for estimating hydraulic properties of the Caribou silt loam soil described by Topp (1971). PSD data and $\theta_{\mathbf{s}}$ for this soil are given in table 2. VG retention parameters calculated from the PSD data are shown in table 3 together with parameter standard errors and the correlation matrix. The retention function, $\theta(h)$, and the relative conductivity function, $K(\theta) / K_{s}$, were calculated from the retention parameters using (2)-(3). In figure 2 , these are compared with the measured retention and conductivity functions as reported by Topp (1971).

Also shown as dashed lines in figure 2 are one-standard-deviation error intervals associated with the predicted functions which were calculated using first-order error analysis. The agreement between measurements and predictions is reasonable for the retention function, with most of the measured data being within one standard deviation of the predicted values. For the relative conductivity function, there is a consistent underprediction using the estimated parameters. This is probably caused by the

Table 3. Estimated vG retention parameters and correlation matrix for example problem.

\begin{tabular}{lccccc}
\hline & & & \multicolumn{3}{c}{ Correlation matrix } \\
\cline { 5 - 7 } Parameter & valimated & $\begin{array}{c}\text { Standard } \\
\text { error }\end{array}$ & $\alpha$ & $n$ & $\theta$, \\
\hline$\alpha\left(\mathrm{cm}^{-1}\right)$ & 0.011 & 0.0072 & 1.000 & & \\
$n$ & 1.169 & 0.0843 & -0.377 & 1.000 & \\
$\theta_{r}$ & $3.8 e-6$ & 0.0832 & -0.595 & 0.875 & 1.000 \\
\hline
\end{tabular}



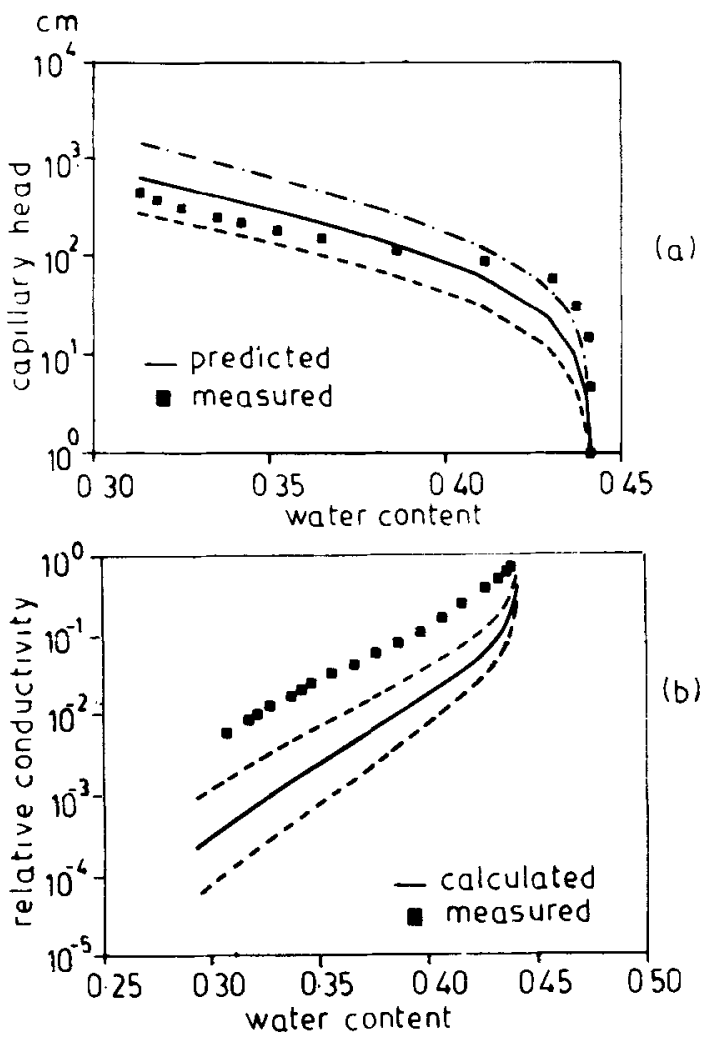

Figure 2. Comparison of measured and predicted retention functions (a) and relative conductivity functions (b). One-standarddeviation error intervals shown as dashed lines.

underprediction of retention data at high water contents, leading to a rapid reduction of relative conductivity near saturated conditions.

Topp (1971) reports a saturated conductivity of $0.598 \mathrm{~cm} \mathrm{~h}^{-1}$ for this soil, whereas (16) predicts a saturated conductivity of $2.04 \mathrm{~cm} \mathrm{~h}^{-1}$, with one-standard-deviation error intervals of $0.135 \mathrm{~cm} \mathrm{~h}^{-1} \leqslant K_{s} \leqslant 30.96 \mathrm{~cm} \mathrm{~h}^{-1}$. The magnitude of these uncertainties suggests that use of (16) as a predictive tool is appropriate for providing order-of-magnitude estimates only.

\section{Effective parameters for simulating flow in heterogeneous systems}

Field-scale simulations of unsaturated flow and transport are complicated by the large degree of spatial variability exhibited by natural soll materials. One approach to deal with soil heterogeneity would be to construct a detailed deterministic description of the physical domain. This would not only pose formidable demands on the amount of data needed to define the system, but also require enormous computational resources. An alternative modelling approach is to treat the actual heterogeneous medium as a fictitious homogeneous system with some set of effective parameters. This necessitates developing procedures for defining effective large-scale properties by scaling-up small-scale parameters.

The behavior of effective medium properties for unsaturated flow in heterogeneous media was studied by Yeh et al (1985) and Mantoglou \& Gelhar (1987) who used simple parametric models to represent soil hydraulic properties as stationary random processes. They applied spectral representation techniques to solve perturbation 
approximations of the governing stochastic differential equations. Expressions for variances of soil-water pressure head, flux and effective hydraulic conductivity were obtained in terms of mean, variance and covariance of the soil properties and mean flow characteristics. It was found that large-scale hysteresis and anisotropy may be caused by spatial variability of medium properties even when local scale hysteresis and anisotropy are disregarded. Yeh et al (1985c) and Mantoglou \& Gelhar (1987c) also noted that anisotropy and heterogeneity of the hydraulic conductivity may induce substantial lateral flow in stratified systems.

Since results of perturbation analyses are strictly applicable to unbounded systems, an alternative is a Monte Carlo simulation approach where the unsaturated flow equation is solved numerically for many equi-probable distributed parameter fields of soil hydraulic properties. Although computationally expensive, the Monte Carlo methodology allows incorporation of boundary effects as well as arbitrary distributions of soil properties. An investigation into the nature of effective parameters for large-scale unsaturated flow is being carried out by Zhu (1989) using Monte Carlo simulation. His approach involves generating distributed fields of soil particle size distribution at every node of the simulation domain as a first step - given mean, variance and autocovariance of local PSD parameters (i.e., mean and standard deviation of the logarithmic CDF). Local PSD data are then converted to local van Genuchten retention parameters ( $\alpha$ and $n$ ) using the procedure described in the previous section. Local values of saturated conductivity are estimated from PSD statistics and porosity using the modified Kozeny-Carman equation, (16). These are input to a finite-element model of unsaturated flow. Local values of $\alpha, n$ and $K_{s}$ estimated in this fashion are found to fit log-normal distributions, which is consistent with field observations (Hopmans et al 1988).

Preliminary simulations have been conducted for steady-state gravity flows. These simulations involve estimating the average volumetric water content and net flux through a two-dimensional vertical system with constant head boundaries at the top and bottom and zero flux conditions at the sides. For several heterogeneous soils, the responses of equivalent homogeneous media, using geometric means for $\alpha, n$ and $K_{s}$ as effective properties, are found to closely reproduce the responses of the actual heterogeneous systems. Figure 3 shows the agreement between retention and conductivity functions of the actual heterogeneous system and the corresponding equivalent homogeneous system for an example soil. The heterogeneous system retention function is derived from the average water content vs. applied capillary head relation, while the conductivity function is derived from the total flux vs. average water content relation. The empirical geometric averaging scheme employed for estimating effective properties appears to work reasonably well in this case.

Because the parameters $\alpha, n$ and $K_{s}$ were found to be log-normally distributed, it is intuitively appealing to use geometric averages as effective properties, particularly for saturated conductivity, in view of perturbation theory results (Gelhar 1986). However, it is interesting to note that the set of effective retention parameters fitted directly to the retention and conductivity functions of the heterogeneous system in figure $3\left(\alpha=0.0178 \mathrm{~cm}^{-1}, n=1.58\right)$ do differ somewhat from the geometric averages $\left(\alpha=0.0125 \mathrm{~cm}^{-1}, n=1.73\right)$ used to compute the equivalent homogeneous system response. Current work is directed at studying the nature of the averaging operator(s) as a function of applied boundary conditions and statistical characteristics of soil property distributions for one- and two-dimensional flows under both steady-state and transient conditions. 

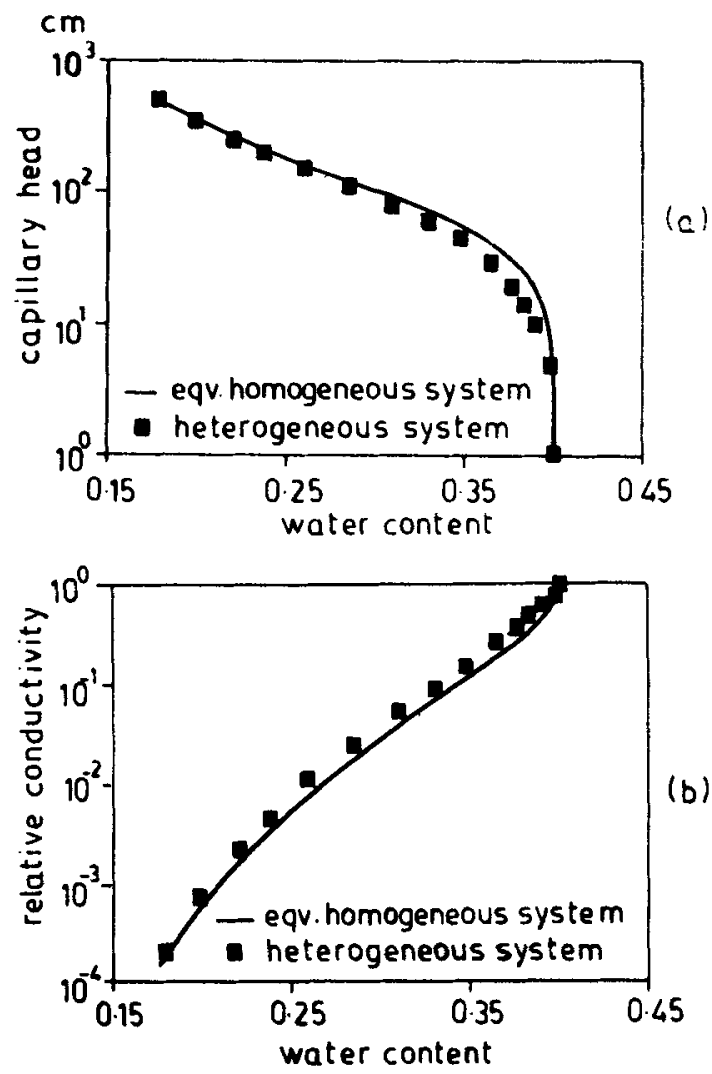

Figure 3. Comparison of retention functions (a) and relative conductivity functions (b) for a heterogeneous system and the equivalent homogeneous system.

\section{Summary and conclusions}

In this paper we have discussed inversion methodology and the use of PSD data for estimating soil hydraulic and transport parameters and demonstrated their applicability to hypothetical and real situations. Issues related to the evaluation of parameter uncertainty and the definition of effective parameters for large-scale simulations in heterogeneous porous media have also been briefly addressed.

Inversion analysis provides a genral framework for the estimation of soil hydraulic and transport properties. Use of a numerical model to solve the parameterized direct problem and a nonlinear optimization scheme to solve the inverse problem facilitates incorporation of arbitrary initial and boundary conditions as well as spatially variable soil properties into the parameter estimation process. This approach ensures that estimated parameters describe the behavior of the porous medium in a manner which is optimal in some specified sense. Another advantage of the optimization approach is that parameter confidence bounds can be quantified, at least approximaely. A disadvantage of the simulation-optimization approach is its computational burden. Estimation of the sensitivity matrix using a forward difference approximation requires the direct problem to be solved at least $N+1$ times (where $N$ is the number of unknown parameters) during every iteration in the optimization phase. Furthermore, the nonlinear nature of the direct problems for unsaturated flow and transport renders the optimization problem highly susceptible to physical factors (i.e., random noise in data, approximations in initial-boundary conditions, etc.) and numerical factors (i.e., convergence criteria, initial guesses, presence of local minima, etc.). Computational 
requirements may be alleviated, however, by using adjoint equations to estimate the sensitivity matrix (e.g., Townley \& Wilson 1985) and by using prior information to constrain parameter estimates to some feasible region.

Use of particle size distribution data for estimating soil hydraulic properties is an appealing alternative because of minimal data requirements and simplicity of the corresponding predictive models. A major problem with using PSD data is the dependence of the model on the calibration data set, i.e., in deriving parameters such as the tortuosity exponent, $\tau$, in the AP model, or the numerical constant in the modified Kozeny-Carman equation, (16). Moreover, since parameter estimates from PSD data are representative only of very small sampling volumes (e.g., core plugs), their application to field-scale simulation models is not straightforward. Nevertheless, both inversion analysis and the use of PSD data should be viewed as complementary methods of parameter estimation, albeit containing information about unsaturated flow and transport at different spatial scales.

Financial support for this study was provided by the Electrical Power Research Institute (Contract RP2485-06), and by the American Petroleum Institute (Contract WM-5-324-7).

\section{References}

Abramowitz M, Stegun I A 1965 Handbook of mathematical functions (New York: Dover)

Arya L M, Paris J F 1981 Soil Sci. Soc. Am., J. 45: 1023-1030

Beck J V, Arnold K J 1977 Parameter estimation in engineering and science (New York: John Wiley and Sons)

Brooks R H, Corey A T 1964 Hydraulic properties of porous media, Hydrology Paper Number 3, Colorado State University, Fort Collins

Carrera J, Neuman S P 1986 Water Resour. Res. 22: 199-210

Dagan G 1986 Water Resour. Res. 22: 120S-134S

Dane J, Hruska S 1983 Soil Sci. Soc. Am. J. 47: 619-624

Dullien F A L 1979 Porous media fluid transport and pore structure (New York: Academic Press)

Freyberg D L 1986 Water Resour. Res. 22: 2031-2046

Gelhar L W 1986 Water Resour. Res. 22: 135S-145S

Gelhar L, W, Mantoglou A, Welty C, Rehfelt K 1985 A review of physical solute transport processes in saturated and unsaturated porous media, Report RP-2485-05, Electrical Power Research Institute, Palo Alto

Haverkamp R, Parlange J Y 1986. Soil Sci. 142: 325-339

Hopmans J W, Schukking H, Torfs P J J F 1988 Water Resour. Res. 24: 2005-2017

Hornung U, Messing W 1982 In Finite elements in water resources P Holz et al (eds.) (Springer Verlag) 18: 15-24

Jury W A, Sposito G 1985 Soil Sci. Soc. Am., J 49: 1331-1341

Jury W A, Stolzy L H, Shouse P A 1982 Water Resour. Res. 18: 369-375

Kool J B, Parker J C 1988 Water Resour. Res. 24: 817-830

Kool J B, Parker J C, van Genuchten M Th 1985 Soil Sci. Soc. Am., J. 49: 1348-1350

Kool J B, Parker J C, van Genuchten M Th 1987 J. Hydrol. 91: 255-293

Lenhard R J, Parker J C, Mishra S 1989 J. Irrig. Drain. Div., Am. Soc. Civ. Eng. 115: 744-751

Mantoglou A, Gelhar L W 1987a Water Resour. Res. 23: 37-46

Mantoglou A, Gelhar L W 1987b Water Resour. Res. 23: 47-56

Mantoglou A, Gelhar L W 1987c Water Resour. Res. 23: 57-67

McCuen R H, Rawls W J, Brakensiek D L 1981 Water Resour. Res. 17: 1005-1013

Mishra S, Parker J C 1989a Water Resour. Res. 25: 385-396 
Mishra S, Parker J C 1989b J. Hydrol. 108: 19-33

Mishra S, Parker J C, Singhal N 1989 J. Hydrol. 108: 1-18

Parker J C and M Th van Genuchten 1984 Determining transport parameters from laboratory and field tracer experiments. Bulletin 84-3, Virginia Agricultural Experiment Station, Virginia Polytechnic Institute and State University, Blacksburg

Rawls W J, Brakensiek D L 1985 Proc. Symposium on Watershed Management (Denver, Co: Am. Soc. Civ. Eng.) pp. 293-299

Topp G E 1971 Water Resour. Res. 7: 914-920

Townley L R, Wilson J L 1985 Water Resour. Res. 21: 1851-1861

van Genuchten M Th 1980 Soil Sci. Soc. Am., J. 44: 892-899

Wagner B W, Gorelick S M 1987 Water Resour. Res. 23: 1162-1174

Yeh T C, Gelhar L W, Gutjahr A L 1985a Water Resour. Res. 21: 447-456

Yeh T C, Gelhar L W, Gutjahr A L 1985b Water Resour. Res. 21: 457-464

Yeh T C, Gelhar L W, Gutjahr A L 1985c Water Resour. Res. 21: 465-471

Yeh W W G 1986 Water Resour. Res. 22: 95-108

Zachmann D W, Duchateau P C, Klute A 1982 Soil Sci. 134: 157-163

Zhu J 1989 Effective properties for unsaturated flow in large scale heterogeneous porous media. Ph D dissertation, Virginia Polytechnic Institute and State University, Blacksburg (in preparation) 